\title{
Comparison of exact and approximate cross-sections in relativistic Coulomb excitation
}

\author{
B.F. Bayman \\ School of Physics and Astronomy, University of Minnesota, \\ 116 Church Street S.E., Minneapolis, MN 55455, U.S.A. \\ and \\ F.Zardi \\ Istituto Nazionale di Fisica Nucleare and Dipartimento di Fisica, \\ Via Marzolo, 8 I-35131 Padova,Italy.
}

November 8, 2018

\begin{abstract}
We present a new method of obtaining time-dependent matrix elements of the electromagnetic pulse produced by a highly-relativistic projectile. These matrix elements are used in a coupled-channel calculation to predict the cross-sections for population of 1- and 2-phonon states of the giant dipole resonance. Comparisons are made with the predictions of the long-wavelength and Born approximations.
\end{abstract}

\section{Introduction}

The subject of relativistic Coulomb excitation has received extensive study in the two decades since its theoretical foundations were established in the classic work of Alder and Winther [1]. In the semiclassical approach to relativistic nuclear Coulomb excitation, the relative motion of the projectile and target is treated classically. Indeed, it is usually assumed to be straight-line motion at constant speed $v$. The evolution of the internal degrees of freedom of each nucleus, under the influence of the classical electromagnetic field produced by the other nucleus, is then followed using quantum mechanics.

There exists a wide literature devoted to the use of relativistic Coulomb excitation for the study of several aspects of nuclear structure that cannot be explored through the nuclear interaction [2-8]. In recent years, this subject has been also shown to be relevant to the practical question of the stability of beams of relativistic heavy ions, since some of the processes which lead to loss of beam ions are initiated by Coulomb excitation [2,9-11].

The situations that are easiest to interpret are those that can be described in terms of the Coulomb excitation of a single level. If this level is unbound, the Coulomb excitation will be followed by particle emission. Methods have been developed to extract 
from the particle emission cross-section the part that is associated with the Coulomb excitation of the emitting state, as opposed to excitation via nuclear forces [12, 13. The Fermi-Williams-Weizsäcker (FWW) method of virtual quanta [14 is frequently used to analyze processes in which Coulomb excitation is followed by particle emission (see, e.g., refs. $[5,12,15,16])$. Here one simulates the electromagnetic pulse by an equivalent flux of virtual photons, and uses experimentally determined photo-nuclear cross-sections to describe the effect of these virtual photons on the target. However, most of the FWW applications to relativistic Coulomb excitation at energies of spectoscopic interest have not used experimentally determined photo-nuclear cross- sections, but have used calculated electromagnetic matrix elements. Thus, in effect, these calculations really amount to the use of the Born approximation as developed in [1], although they are expressed in the language of the method of virtual photons.

Analyses of experimental data based on the Born approximation imply that the final state is weakly coupled to all states other than to the initial state. The Born approximation is just the first term of the Born (or Fredholm) series, which constitutes a formal solution of the time-dependent Schrödinger equation. In dealing with multi-channel processes, some workers [17] have attempted to include a few higher terms of this series. Other approaches, based on coupled-channel methods, [5, 6], have attempted a numerical solution of the Schrödinger equation which, in principle, sums all terms of the Born series. The harmonic vibrator method allows for complete coupling of all the states of a vibrational band, if some simplifying assumptions are made concerning the ratios of interaction matrix elements.

All these calculations require matrix elements of the electromagnetic interaction between the nuclei, defined with respect to the eigenstates of internal motion. If the relative velocity of the nuclei is relativistic, the interaction is strongly retarded, which introduces difficulties into the evaluation of its matrix elements. The long- wavelength approximation (LWLA) has proven to be a very useful device for overcoming these difficulties [5, 6].

We see then that two approximations have been extensively used in this field: the Born approximation to solve the Schrödinger equation and the long-wavelength approximation to evaluate matrix elements. These approximations have been found to be effective below bombarding energies of $2 \mathrm{GeV}$ per nucleon, which has been adequate for most nuclear structure investigations done so far. At higher energies, which could be of interest in future studies, and for energies presently considered in colliders, these approximations may not be adequate.

Much of the recent effort in the theoretical study of relativistic Coulomb excitation is devoted to the nuclear structure aspects of the problem. For example, in the study of giant resonances, one must take account of the spreading of the resonance amongst the background states (see, e.g., refs. 15, 8, 18, 19, 20, 21]), and the anharmonicity of the oscillation 4, 6, 22, 23, 24. Of course, these studies also must incorporate the interaction of the nuclear motion with the electromagnetic pulse, and this is generally done by means of the Born approximataion and/or the the LWLA. For this reason, we believe it is important to understand the limitations of these approximations, even in Coulomb excitation studies whose primary emphasis is on nuclear structure.

The purpose of this article is to assess the ranges of validity of the LWLA and the Born approximation. We accomplish this by comparison with a general numerical solution 
of the time-dependent Schrödinger equation, expressed as a set of coupled differential equations. The energy range considered here extends from energies currently used for nuclear structure studies (see, e.g. refs. $[5,6]$ ), up to about $10 \mathrm{GeV}$ per nucleon. The methods employed are quite general, and could be used at still higher energy.

In Section 2, a general survey of methods and approximations is given. In Section 3, we present our method for numerical evaluation of the Fourier transform of interaction matrix elements. In Sections 4 and 5, we illustrate our procedure by investigating the relativistic Coulomb excitation of giant dipole resonance (GDR) phonons. We present numerical results for the particular case of ${ }^{208} \mathrm{~Pb}$ projectiles exciting GDR states of ${ }^{40} \mathrm{Ca}$. These results are compared with those produced by approximate approaches. We summarize in Section 6 the main results we obtained.

\section{Survey of methods used in RCE}

In the semiclassical theory of relativistic Coulomb excitation, the electromagnetic field between the projectile and target is treated classically, but their internal degrees of freedom are treated according to the principles of quantum mechanics. The time dependent Schroedinger equation is written

$$
i \hbar \frac{\partial \psi(t)}{\partial t}=\left[H_{0}+V(t)\right] \psi(t)
$$

with $H_{0}$ referring only to the target ${ }^{1}$ degrees of freedom, and $V(t)$ the interaction between the target and the electromagnetic field of the projectile:

$$
V(t)=\int\left[\varphi_{C}^{\mathrm{ret}}\left(\mathbf{r}^{\prime}, t\right) \rho\left(\mathbf{r}^{\prime}\right)-\frac{1}{c} \mathbf{A}_{C}^{\mathrm{ret}}\left(\mathbf{r}^{\prime}, t\right) \cdot \mathbf{j}\left(\mathbf{r}^{\prime}\right)\right] d^{3} r^{\prime}
$$

The scalar and the vector potentials associated with the projectile electromagnetic field are $\varphi_{C}^{\mathrm{ret}}\left(\mathbf{r}^{\prime}, t\right), \mathbf{A}_{C}^{\mathrm{ret}}\left(\mathbf{r}^{\prime}, t\right)$, and $\rho\left(\mathbf{r}^{\prime}\right), \mathbf{j}\left(\mathbf{r}^{\prime}\right)$ are the target transition charge and current densities, respectively.

The projectile is assumed to have a spherically symmetric charge distribution, with total charge $Z_{P} e$. Because of its large momentum, it can be assumed to follow a straightline trajectory at constant speed $v$. Thus its center is located at time $t$ by

$$
\mathbf{r}=b \hat{\mathbf{y}}+v t \hat{\mathbf{z}}
$$

The constant $b$ is the impact parameter. Excitation probabilities are calculated as functions of $b$. The scalar and vector potentials of the projectile field, at the target point $\mathbf{r}^{\prime}$, are given by 14

$$
\begin{aligned}
\varphi_{C}^{\mathrm{ret}}\left(\mathbf{r}^{\prime}, t\right) & =\frac{Z_{P} e \gamma}{\sqrt{x^{\prime 2}+\left(y^{\prime}-b\right)^{2}+\gamma^{2}\left(z^{\prime}-v t\right)^{2}}} \\
\mathbf{A}_{C}^{\mathrm{ret}}\left(\mathbf{r}^{\prime}, t\right) & =\frac{v}{c} \varphi_{C}^{\mathrm{ret}}\left(\mathbf{r}^{\prime}, t\right) \hat{\mathbf{z}}
\end{aligned}
$$

\footnotetext{
${ }^{1}$ For simplicity, we restrict our discussion to the situation in which only the target is excited, but the argument is easily generalized to allow for projectile excitation as well.
} 
Because of the factor $\gamma^{2}$ in the denominator of Eq.(2.4), these potentials are not spherically symmetric about the projectile center.

The Schroedinger equation (2.1) is conveniently expressed in terms of an expansion in eigenstates $\phi_{\alpha}$ of the nuclear Hamiltonian $H_{0}$,

$$
\psi(t)=\sum_{\alpha} a_{\alpha}(t) e^{\frac{i}{\hbar} \epsilon_{\alpha} t} \phi_{\alpha}
$$

with

$$
\begin{aligned}
H_{0} \phi_{\alpha} & =\epsilon_{\alpha} \phi_{\alpha}, \quad<\phi_{\alpha} \mid \phi_{\beta}>=\delta_{\alpha \beta} ; \\
V_{\alpha \beta}(t) & =<\phi_{\alpha}\left|\varphi_{C}^{\mathrm{ret}}(t) \rho-\frac{1}{c} \mathbf{A}_{C}^{\mathrm{ret}}(t) \cdot \mathbf{j}\right| \phi_{\beta}>.
\end{aligned}
$$

If (2.5) is substituted into (2.1), the result is a set of coupled ordinary differential equations for the amplitudes $a_{\alpha}(t)$ :

$$
i \hbar \frac{d a_{\beta}(t)}{d t}=\sum_{\alpha} e^{\frac{i}{\hbar}\left(\epsilon_{\beta}-\epsilon_{\alpha}\right) t} V_{\beta \alpha}(t) a_{\alpha}(t) .
$$

These equations must be solved subject to initial conditions

$$
a_{\alpha}(-\infty)=\delta_{\alpha, 0}
$$

The probability that the target will be in state $\phi_{\beta}$ after the collision is given by $\left|a_{\beta}(+\infty)\right|^{2}$, and the cross-section for the population of $\phi_{\beta}$ is given by

$$
\sigma_{\beta}=\int_{b_{\min }}^{\infty} 2 \pi\left|a_{\beta}(+\infty)\right|^{2} b d b
$$

Here $b_{\min }$ is usually taken to be somewhat larger than the sum of the target and projectile radii 3 .

The first step in the solution of Eq.(2.8) is the computation of the interaction matrix elements $V_{\beta \alpha}(t)$. This is a formidable task, since the retardation of the interaction introduces a directional asymmetry, which means that the interaction is not invariant under rotations of the internal coordinates alone. Fortunately, Alder and Winther have found a convenient multipole expansion of the Fourier transform of the $V_{\beta \alpha}(t)$,

$$
V_{\beta \alpha}(\omega) \equiv \int_{-\infty}^{\infty} \frac{d t}{\hbar} e^{i \omega t} V_{\beta \alpha}(t)
$$

The $V_{\beta \alpha}(\omega)$ defined in Eq.(2.10) can be used directly in a coupled integral equation formulation of the Schroedinger equation, as was done in ref. 25]. However this approach is difficult to implement when many states $\phi_{\alpha}$ have to be included in the calculation. Another alternative is to proceed by inverting the Fourier transform (2.10)

$$
V_{\beta \alpha}(t)=\frac{\hbar}{2 \pi} \int_{-\infty}^{\infty} d \omega e^{-i \omega t} V_{\beta \alpha}(\omega)
$$

to convert the $V_{\beta \alpha}(\omega)$ obtained from the Alder- Winther expansion into $V_{\beta \alpha}(t)$ which can be used in the time- dependent formulation (2.8) of the Schroedinger equation. 
There is no known closed expression for the Fourier transform (2.11). In ref. [6] Lanza et al replaced the exact Alder-Winther expression for $V_{\beta \alpha}(\omega)$ by its long-wavelength approximation (LWLA). Here one assumes that the important Fourier components of the electromagnetic pulse correspond to wavelengths that are large compared to target dimensions. In this case, the small-argument limit of the spherical Bessel function,

$$
j_{\lambda}\left(\frac{\omega}{c} r^{\prime}\right) \sim \frac{\left(\frac{\omega}{c} r^{\prime}\right)^{\lambda}}{(2 \lambda+1) ! !},
$$

can be used. With this approximation, Lanza et al [6] were able to obtain explicit approximate expressions, in terms of hypergeometric functions, for the $V_{\beta \alpha}(t)$, which they used in their analyses of multiphonon Coulomb excitation. They tested the validity of the LWLA by comparing a few $V_{\beta \alpha}(t)$ calculated using the LWLA with numerical evaluations of the Fourier transform (2.11) of the exact $V_{\beta \alpha}(\omega)$. They concluded that the LWLA was adequate for their analysis of the ${ }^{208} \mathrm{~Pb}+{ }^{208} \mathrm{~Pb}$ collision at $\mathrm{E}_{\text {lab }}=641 \mathrm{MeV}$.

Bertulani et al [5] approached the problem of finding approximate $V_{\beta \alpha}(t)$ by expressing the potential (2.4) in a Taylor expansion around $x^{\prime}=y^{\prime}=z^{\prime}=0$. This formally exact expansion was truncated in a manner that caused the remaining terms to be precisely equal to those given by the LWLA expressions in ref. [6]. However, the advantage of the truncated Taylor expansion is that it yields simple expressions for the $V_{\beta \alpha}(t)$ which, although they are equal to the hypergeometric functions used in ref. [6], are much more transparent. The general proof of the equivalence of these methods can be found in ref. [26].

The approach to be followed in this manuscript is the use of a quick and accurate method for the numerical evaluation of the Fourier tranform $(2.11)$, using exact $V_{\beta \alpha}(\omega)$. The $V_{\beta \alpha}(t)$ calculated in this way will be used in the numerical solution of the coupled time-dependent equations (2.8). We will thus be able to obtain essentially exact Coulombexcitation cross-sections at any bombarding energy. These exact cross-sections can be used to explore the limits of validity of the LWLA and other approximations.

\section{Numerical Evaluation of the Fourier transform}

The difficulty of evaluating (2.11) as a numerical integral is the rapid oscillation of the integrand for high values of $t$. Our approach to this problem is a generalization of the idea behind the use of Simpson's rule in the evaluation of $\int_{x_{i}}^{x_{f}} f(x) d x$. We first divide the $\omega$-integration range of (2.11) into an even number of steps of length $h$. We assume that these steps are small enough so that, over every adjacent pair of steps, $V_{\beta \alpha}(\omega)$ can be approximated by a quadratic function of $\omega$. Thus for the interval $\omega_{1}-h \leq \omega \leq \omega_{1}+h$, we make the approximation

$$
V_{\beta \alpha}(\omega) \sim\left(\frac{\omega-\omega_{1}}{h}\right)^{2} u+\left(\frac{\omega-\omega_{1}}{h}\right) v+w
$$

We can make this approximation exact at $\omega=\omega_{1}, \omega_{1} \pm h$ by defining $u, v$, and $w$ to be

$$
u=\frac{V_{\beta \alpha}\left(\omega_{1}-h\right)+V_{\beta \alpha}\left(\omega_{1}+h\right)}{2}-V_{\beta \alpha}\left(\omega_{1}\right)
$$




$$
\begin{aligned}
v & =\frac{V_{\beta \alpha}\left(\omega_{1}+h\right)-V_{\beta \alpha}\left(\omega_{1}-h\right)}{2} \\
w & =V_{\beta \alpha}\left(\omega_{1}\right) .
\end{aligned}
$$

Note that since the quadratic approximation is applied to $V_{\beta \alpha}(\omega)$, its validity is independent of $t$. Then the $\omega_{1}-h \leq \omega \leq \omega_{1}+h$ part of the integration (2.11) can be approximated by

$$
\int_{\omega_{1}-h}^{\omega_{1}+h} d \omega e^{-i \omega t} V_{\beta \alpha}(\omega) \sim \int_{\omega_{1}-h}^{\omega_{1}+h} d \omega e^{-i \omega t}\left[\left(\frac{\omega-\omega_{1}}{h}\right)^{2} u+\left(\frac{\omega-\omega_{1}}{h}\right) v+w\right]
$$

Now an exact integration of the right-hand side of (3.1) yields

$$
\begin{gathered}
\int_{\omega_{1}-h}^{\omega_{1}+h} d \omega e^{-i \omega t} V_{\beta \alpha}(\omega) \simeq \\
\frac{2}{h^{2} t^{3}}\left\{h t \cos (h t)\left(2 u \cos \left(\omega_{1} t\right)+v h t \sin \left(\omega_{1} t\right)\right)\right. \\
\left.+\sin (h t)\left(\left(-2 u+h^{2} t^{2}(u+w)\right) \cos \left(\omega_{1} t\right)-v h t \sin \left(\omega_{1} t\right)\right)\right\} \\
-\frac{2 i}{h^{2} t^{3}}\left\{h t \cos (h t)\left(2 u \sin \left(\omega_{1} t\right)-v h t \cos \left(\omega_{1} t\right)\right)\right. \\
\left.+\sin (h t)\left(\left(-2 u+h^{2} t^{2}(u+w)\right) \sin \left(\omega_{1} t\right)+v h t \cos \left(\omega_{1} t\right)\right)\right\}
\end{gathered}
$$

This approximation is used for every adjacent pair of steps in the $\omega$ integration.

Equation (3.2) cannot be used at $t=0$, since it takes the form $0 / 0$ there. By expanding the quantities between \{\} about $t=0$, we can show that

$$
\begin{gathered}
\lim _{t \rightarrow 0} \int_{\omega_{1}-h}^{\omega_{1}+h} d \omega e^{-i \omega t} V_{\beta \alpha}(\omega) \\
\simeq \frac{2}{3} h(u+3 w)-\frac{t^{2}}{15}\left(h^{2}(3 u+5 w)+10 h v \omega_{1}+5(u+3 v) \omega_{1}^{2}\right) \\
-\frac{2 i}{3} h t\left(h v+(u+3 w) \omega_{1}\right)
\end{gathered}
$$

The only circumstance where the quadratic representation of $V_{\beta \alpha}(\omega)$ is inadequate occurs when $V_{\beta \alpha}(\omega)$ is singular at $\omega=0$. In these cases $V_{\beta \alpha}(\omega)$ has the form

$$
V_{\beta \alpha}(\omega)=f(\omega) K_{0}\left(\frac{|\omega| b}{\gamma v}\right)
$$

with $f(0) \neq 0$. Since $K_{0}\left(\frac{|\omega| b}{\gamma v}\right)$ diverges logarithmically as $\omega \rightarrow 0$, numerical integration of (3.4) requires special precautions. In this case, we work with the identity

$$
V_{\beta \alpha}(\omega)=(f(\omega)-f(0)) K_{0}\left(\frac{|\omega| b}{\gamma v}\right)+f(0) K_{0}\left(\frac{|\omega| b}{\gamma v}\right) .
$$


The first term in (3.5) is regular at $\omega=0$, and its Fourier transform can be evaluated without difficulty using Eqs.(3.2) and (3.3). The Fourier transform of the second term in (3.5) can be evaluated exactly

$$
\frac{\hbar}{2 \pi} \int_{-\infty}^{\infty} d \omega e^{-i \omega t} f(0) K_{0}\left(\frac{|\omega| b}{\gamma v}\right)=\frac{f(0) \hbar v}{2 \sqrt{\left(\frac{b}{\gamma}\right)^{2}+(v t)^{2}}}
$$

This term dominates the interaction matrix element $V_{\beta \alpha}(t)$ at large values of $|t|$.

\section{Brink's model for the Giant Dipole Resonance}

\subsection{The GDR phonon states}

The giant dipole resonance can be regarded as a collective oscillation of the protons in a nucleus relative to the neutrons [27]. The one-phonon GDR state also has a simple interpretation in terms of an isovector linear combination of one-particle, one-hole excitations of the ground state 28. It was shown by Brink 29] that states of the GDR have a simple interpretaion within the harmonic oscillator shell model, in terms of harmonic oscillations of the vector $\mathbf{R}_{\mathrm{pn}}$ connecting the centers of unexcited proton and neutron spheres. We use this representation of the GDR to provide the transition charge and current densities needed for the calculation of the $V_{\beta \alpha}(\omega)$ (see App. A).

An individual GDR state is described by the three quantum numbers $N, L, M$, in which $N(=0,1,2 \cdots)$ is the principal quantum number. The number of GDR phonons associated with this state is $2 N+L$. Thus the $\beta, \alpha$ in $V_{\beta \alpha}(\omega)$ stand for $N_{\beta}, L_{\beta}, M_{\beta}$ and $N_{\alpha}, L_{\alpha}, M_{\alpha}$, respectively. These states are denoted $\Psi_{M}^{N L}$.

\subsection{Structure of the matrix elements}

The algebraic details of the construction of the GDR states, and the derivations of the formulae for the associated $V_{\beta \alpha}(\omega)$, are given in the Appendices. In order to facilitate our discussion of the long-wavelength approximation, and of the features that lead to convergence of the $\omega$ integral, we summarize here the general structure of the result.

$V_{\beta \alpha}(\omega)$ can be expressed in terms of the Fourier transforms of the scalar and vector potentials as follows:

$V_{\beta \alpha}(\omega)=\left\langle\Psi_{M_{\beta}}^{N_{\beta} L_{\beta}}|V(\omega)| \Psi_{M_{\alpha}}^{N_{\alpha} L_{\alpha}}>=\int d^{3} r^{\prime}\left[\rho_{\beta \alpha}\left(\mathbf{r}^{\prime}\right) \varphi_{C}^{\mathrm{ret}}\left(\mathbf{r}^{\prime}, \omega\right)-\frac{1}{c} \mathbf{j}_{\beta \alpha}\left(\mathbf{r}^{\prime}\right) \cdot \mathbf{A}_{C}^{\mathrm{ret}}\left(\mathbf{r}^{\prime}, \omega\right)\right]\right.$,

where

$$
\begin{gathered}
\varphi_{C}^{\mathrm{ret}}\left(\mathbf{r}^{\prime}, \omega\right)=\int_{-\infty}^{\infty} \frac{d t}{\hbar} e^{i \omega t} \varphi_{C}^{\mathrm{ret}}\left(\mathbf{r}^{\prime}, t\right) \\
\mathbf{A}_{C}^{\mathrm{ret}}\left(\mathbf{r}^{\prime}, \omega\right)=\frac{v}{c} \varphi_{C}^{\mathrm{ret}}\left(\mathbf{r}^{\prime}, \omega\right) \hat{\mathbf{z}}
\end{gathered}
$$

The multipole expansion of $\varphi_{C}^{\mathrm{ret}}\left(\mathbf{r}^{\prime}, \omega\right)$, given first by Alder and Winther [1], can be written in the form

$$
\varphi_{C}^{\mathrm{ret}}\left(\mathbf{r}^{\prime}, \omega\right)=\sum_{\lambda, \mu} K_{\mu}\left(\frac{|\omega| b}{\gamma v}\right) C_{\lambda, \mu}(\omega) j_{\lambda}\left(\frac{|\omega|}{c} r^{\prime}\right) Y_{\mu}^{\lambda}\left(\hat{\mathbf{r}}^{\prime}\right)
$$


with

$$
C_{\lambda, \mu}(\omega) \equiv e^{-i \mu \frac{\pi}{2}} \frac{2 Z_{P} e}{\hbar v} \mathcal{G}_{\lambda, \mu}
$$

where

$$
\mathcal{G}_{\lambda, \mu}=\frac{i^{\lambda+\mu}}{(2 \gamma)^{\mu}}\left(\frac{\omega}{|\omega|}\right)^{\lambda-\mu}\left(\frac{c}{v}\right)^{\lambda} \times \sqrt{4 \pi(2 \lambda+1)(\lambda-\mu) !(\lambda+\mu) !} \sum_{n} \frac{1}{(2 \gamma)^{2 n}(n+\mu) ! n !(\lambda-\mu-2 n) !}
$$

It is shown in Appendix B that the $\int \varphi \rho$ part of the matrix element can be expressed in the form:

$$
\begin{gathered}
\int d^{3} r^{\prime} \varphi_{C}^{\mathrm{ret}}\left(\mathbf{r}^{\prime}, \omega\right) \rho_{\beta \alpha}\left(\mathbf{r}^{\prime}\right)=\sum_{\lambda \mu} C_{\lambda, \mu}(\omega) K_{\mu}\left(\frac{|\omega| b}{\gamma v}\right) \int d^{3} r^{\prime} \rho_{\beta \alpha}\left(\mathbf{r}^{\prime}\right) j_{\lambda}\left(\frac{|\omega|}{c} r^{\prime}\right) Y_{\mu}^{\lambda}\left(\hat{r}^{\prime}\right) \\
\simeq e^{\frac{(\omega / c)^{2}}{8 T_{T^{\nu}}}} \sum_{n^{\prime}, \ell^{\prime}} 2\left(2 \ell^{\prime}+1\right) \int_{0}^{\infty} j_{0}\left(\frac{\omega}{c} r^{\prime}\right) u_{n^{\prime}, \ell^{\prime}}^{2}\left(r^{\prime}\right) r^{\prime 2} d r^{\prime} \sum_{n \ell N L} A_{n \ell N L} K_{\mu}\left(\frac{|\omega| b}{\gamma v}\right) \tilde{u}_{N L}\left(\frac{\omega / c}{2} \sqrt{2}\right),
\end{gathered}
$$

where $A_{n \ell N L}$ are suitable coefficients. The functions $u_{n \ell}(r)$ are the harmonic oscillator radial functions associated with the individual nucleons moving in the shell-model potential,

$u_{n \ell}(r)=\sqrt{\frac{2^{\ell-n+2}(2 \ell+2 n+1) ! ! \nu^{3 / 2}}{\sqrt{\pi} n !}}(\sqrt{\nu} r)^{\ell} e^{-\frac{\nu r^{2}}{2}} \times \sum_{\kappa} \frac{(-2)^{\kappa} n !}{\kappa !(n-\kappa) !(2 \ell+2 \kappa+1) ! !}\left(\nu r^{2}\right)^{\kappa}$,

where $\nu=m \omega_{\mathrm{sm}} / \hbar$, with $\omega_{\mathrm{sm}}$ representing the frequency associated with the shell-model harmonic oscillator potential. The tilde in the corresponding Fourier transformed states $\tilde{u}$ signifies that the size parameter is taken to be $2 /\left(Z_{T} \nu\right)$. The matrix elements of the $-\frac{1}{c} \int \mathbf{j}_{\beta \alpha} \cdot \mathbf{A}_{C}^{\text {ret }}$ part of the electromagnetic interaction are easily represented in terms the above formulae (see Appendix C).

The advantage of this approach is that it yields explicit functions for the matrix elements $V_{\beta \alpha}(\omega)$, with only the single parameter $\nu$ (which is related to the nuclear radius). Of course, the representation of GDR states in terms of eigenstates of the independent particle shell model with oscillator radial functions is highly schematic. Some important features of the real GDR, such as the spreading of the GDR among background states, are missing from this model. However, the states we use give a realistic picture of the collective oscillation of the nuclear protons and neutrons relative to each other, and we believe they provide sufficiently accurate $V_{\beta \alpha}(\omega)$ to enable us to test the dynamics of the Coulomb excitation process.

\subsection{The Long-Wavelength Approximation (LWLA)}

\subsubsection{Expression of the LWLA in Brink's model of the GDR}

We can get the result of using the long wavelength approximation (2.12) in (4.4) by keeping only the lowest powers of $\frac{|\omega|}{c}$ in (4.5), apart from the $\omega$ dependence of $K_{\left|M_{\beta}-M_{\alpha}\right|}\left(\frac{|\omega| b}{\gamma v}\right)$. This implies the following replacements in (4.5):

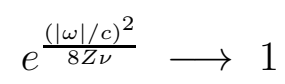




$$
\begin{gathered}
\int_{0}^{\infty} j_{0}\left(\frac{|\omega|}{c} r^{\prime}\right) u_{n, \ell}^{2}\left(r^{\prime}\right) r^{\prime 2} d r^{\prime} \longrightarrow \int_{0}^{\infty} u_{n, \ell}^{2}\left(r^{\prime}\right) r^{\prime 2} d r^{\prime}=1 \\
\tilde{u}_{N L}\left(\frac{|\omega| / c}{2 \sqrt{2}}\right) \longrightarrow \sqrt{\frac{2^{L-N+2}(2 L+2 N+1) ! !}{(Z \nu / 2)^{3 / 2} \sqrt{\pi}[(2 L+1) ! !]^{2}}}\left(\frac{|\omega| / c}{2 \sqrt{Z \nu}}\right)^{L}
\end{gathered}
$$

If these replacements are made, our calculated results are equal to those obtained with the LWLA. In Section 5 we will test the adequacy of the long-wavelength approximation, for varying bombarding energies and impact parameters.

\subsubsection{General conditions for the validity of the LWLA}

If the integral representation $(2.11)$ of $V_{\beta \alpha}(t)$ is to converge, it is necessary that $V_{\beta \alpha}(\omega)$ should decrease sufficiently rapidly as $|\omega| \rightarrow \infty$. We use the term damping to refer to the phenomena responsible for this decrease. It is evident from (4.4) that the decrease of $V_{\beta \alpha}(\omega)$ with increasing $|\omega|$ has two causes:

1) Kinematic damping. The factor $K_{\mu}\left(\frac{|\omega| b}{\gamma v}\right)$ has a large- $|\omega|$ limit of

$$
K_{\mu}\left(\frac{|\omega| b}{\gamma v}\right) \stackrel{|\omega|>>\frac{\gamma v}{b}}{\longrightarrow} \sqrt{\frac{\pi}{2\left(\frac{|\omega| b}{\gamma v}\right)}} e^{-\frac{|\omega| b}{\gamma v}}
$$

This decrease occurs because the time-width of the electromagnetic pulse at the target is of the order of $\left(\frac{b / \gamma}{v}\right)$, which contains frequency components up to, but not greatly exceeding, $\omega_{\max } \sim \frac{\gamma v}{b}$. It can also be said that the impulse is too adiabatic to contain frequency components with $\omega>\omega_{\max }$.

2) Dynamic damping. If $\frac{|\omega|}{c} R>>1$, the factor $j_{\lambda}\left(\frac{|\omega|}{c} r^{\prime}\right)$ in (4.4) will go through many oscillations as $r^{\prime}$ is integrated from 0 to $R$. These oscillations will lead to cancellations between different parts of the $r^{\prime}$ integration range, which will lead to a decrease of $V_{\beta \alpha}(\omega)$ when $|\omega|>c / R$. This phenomena can also be called retardation damping, because it is associated with the finite speed, $c$, of propagation of electromagnetic signals. Because of this finite speed, different parts of the target nucleus, at one time $t$, are influenced by the projectile at different points along its orbit. This implies that the $r^{\prime}$ integration needed for the evaluation of $V_{\beta \alpha}(\omega)$ effectively produces a time average over the projectile history, which smooths out sharper features of this history. Thus $V_{\beta \alpha}(t)$ will not vary as rapidly with $t$ as it would if there had been no retardation. Equivalently, the presence of high- $|\omega|$ components in $V_{\beta \alpha}(\omega)$ is diminished.

It is worth remarking that the criterion for dynamic damping, $|\omega|>c / R$, is opposite to the validity condition for the LWLA, $|\omega|<c / R$. Thus if we remain within the regime of validity of the LWLA, we will not experience dynamic damping; in this regime, the only phenomenon that can lead to the damping required for the convergence of the integral (2.11) is kinematic damping. Hence we conclude that the LWLA can only be applied if $|\omega|<c / R$ for the entire $|\omega|$-range from $\omega=0$ up to $\omega_{\max } \sim \frac{\gamma v}{b}$, the frequency at which kinematic damping becomes effective. This implies that

$$
\omega_{\max } \sim \frac{\gamma v}{b}<\frac{c}{R}
$$




$$
\frac{b}{R}>\frac{\gamma v}{c}=\sqrt{\gamma^{2}-1}
$$

must be true if the LWLA is to be trusted.

\section{Numerical results in ${ }^{40} \mathbf{C a}-{ }^{208} \mathbf{P b}$ collisions}

\subsection{Comparison of excitation amplitudes}

The coupled differential equations (2.8) for the $a_{\beta}(t)$ were numerically integrated using the fourth-order Runge-Kutta method. We have included all states with $\leq$ two phonons, i.e. the states with $(N L M)=(000),(010),(011),(020),(021),(022),(100)(M=1,2$ here represents the reflection-symmetric combinations of the $M= \pm 1, \pm 2$ states.) A test of the numerical accuracy of the integration procedure is the extent to which the total normalization of the state, $\sum_{\beta}\left|a_{\beta}(t)\right|^{2}=1$, is preserved. It was not difficult to satisfy this condition to 8 decimal places, even in situations when there was strong coupling between the different $a_{\beta}(t)$.

The only free parameter in our model is the size parameter, $\nu=m \omega_{\mathrm{sm}} / \hbar$, that characterizes the shell-model potential. For simplicity, we follow the usual prescrition

$$
\hbar \omega_{\mathrm{sm}} \sim 40 A^{-\frac{1}{3}} \mathrm{MeV}
$$

which, in the case of ${ }^{40} \mathrm{Ca}$, leads to $\hbar \omega_{\mathrm{sm}}=11.7 \mathrm{MeV}, \nu=.28177 \mathrm{fm}^{-2}$.

We illustrate the criterion of validity of the LWLA with the example of ${ }^{208} \mathrm{~Pb}$ projectiles of kinetic energy $10 \mathrm{~A} \mathrm{GeV}$, on a ${ }^{40} \mathrm{Ca}$ target. Then $\gamma=1+10 / .938 \sim 11.66$, and the condition of validity of LWL is

$$
b>\sqrt{(11.66)^{2}-1} \times R \sim 50 \mathrm{fm} .
$$

Figures 1a and $1 \mathrm{~b}$ show $V_{\beta \alpha}(\omega)$ and $V_{\beta \alpha}(t)$ for $b=12 \mathrm{fm}$, with $\alpha$ referring to the ground state and $\beta$ referring to the one-phonon state with $M=1$. It is evident that the exact expression for $V_{\beta \alpha}(\omega)$, which includes dynamic as well as kinematic damping, has less high- $|\omega|$ content than does the LWLA expression. Correspondingly, $V_{\beta \alpha}(t)$ calculated with the LWLA is more sharply peaked than is the exact $V_{\beta \alpha}(t)$. Figures $2 \mathrm{a}$ and $2 \mathrm{~b}$ show the same comparison, but for $b=50 \mathrm{fm}$, where the above argument suggests that the LWLA should be adequate. While it is clear that the LWLA does a better job at $b=50$ $\mathrm{fm}$ than at $b=12 \mathrm{fm}$, the approximation at $b=50 \mathrm{fm}$ is still only fair.

The calculation of the total excitation probability requires an integration over $b$. Thus the inadequacy of the LWLA for small $b$ will have serious consequences only if the small- $b$ part of the integration range makes an important contribution to the total $b$-integration. We will see in Section 5 that the bombarding energy determines whether or not this occurs.

Some typical plots of $P_{\beta}(t) \equiv\left|a_{\beta}(t)\right|^{2}$ are shown in Figures 3 through 6 .

Figure 3 shows the occupation probability of $\Psi_{1}^{01}$ for an impact parameter of $200 \mathrm{fm}$. The horizontal line indicates the Born approximation prediction. It is seen that, beginning near $t=0 \mathrm{fm} / \mathrm{c}$, the occupation probability rises almost monotonically to the Born value, and then remains there. The occupation probability of $\Psi_{0}^{01}$ shown in Figure 4 has a very

different behavior. The coupling between $\Psi_{0}^{00}$ and $\Psi_{0}^{01}$ has a long range, because the 
$\lambda=\mu=0$ term in the $\mathbf{j} \cdot \mathbf{A}$ part of the interaction can connect these states. It is seen in Figure 4 that the occupation probability rises to a maximum near $t=0 \mathrm{fm} / \mathrm{c}$, and then strongly decreases. The asymptotic probability in this case is $0.6 \times 10^{-5}$, compared to the Born prediction of $\sim 10^{-8}$. The rise and fall of the $\Psi_{0}^{01}$ occupation probability is associated with the fact that $\Psi_{0}^{01}$ represents a longitudinal oscillation (with reference to the direction of the projectile motion), and the coupling to the ground state changes sign during the encounter. $\Psi_{1}^{01}$, on the other hand, is a transverse oscillation, and the coupling to the ground state has a constant sign.

The Born prediction for the excitation probablility of $M=0$ states is always very small at high bombarding energy (see Section 5.3.1 below).

The corresponding curves for a grazing collison $(b=12 \mathrm{fm})$ are shown in Figures 5 and 6. Again the $\Psi_{1}^{01}$ occupation probability rises almost monotonically to a constant value, but in this case the constant value is significantly less than the Born probability. The population of $\Psi_{0}^{01}$ shows a rather complicated time structure. The asymptotic occupation probability is again much greater than the Born probability.

The small-amplitude oscillation of the occupation probability of $\Psi_{0}^{01}$ shown in Figure 6 for $b=12 \mathrm{fm}$ and $t>100 \mathrm{fm} / \mathrm{c}$ is a consequence of the long range of the $\lambda=\mu=0$ coupling term.

\subsection{Evaluation of the cross section}

Figures 7 and 8 show the asymptotic probabilites for $\Psi_{0}^{01}$ and $\Psi_{1}^{01}$, plotted as functions of impact paramater $b$.

In the $\Psi_{1}^{01}$ case, it is seen that the Born expression gives a very good representation of the exact probability when $b \gtrsim 100 \mathrm{fm}$. In this situation, the most convenient way to evaluate the cross-section is to break the impact-parameter integral (2.9) into two terms:

$$
\sigma_{\beta}=\int_{b_{\min }}^{b^{\prime}} 2 \pi\left|a_{\beta}(+\infty)\right|^{2} b d b+\int_{b^{\prime}}^{\infty} 2 \pi\left|a_{\beta}(+\infty)\right|^{2} b d b
$$

where $b^{\prime} \gtrsim 100 \mathrm{fm}$. The first integral is evaluated numerically using $\left|a_{\beta}(+\infty)\right|^{2}$ calculated by integrating the Schrodinger equation for a range of $b$ values between $b_{\text {min }}$ and $b^{\prime}$. The second integral can be calculated exactly, because the $b$ dependence of the Born probability is given by $\left(K_{\mu}\left(\frac{|\omega| b}{\gamma v}\right)\right)^{2}$, and one can take advantage of the exact formula

$$
\int_{\xi}^{\infty}\left(K_{\mu}(x)\right)^{2} x d x=\frac{\xi^{2}}{2}\left[\left(K_{\mu+1}(\xi)\right)^{2}-\left(K_{\mu}(\xi)\right)^{2}-\frac{2 \mu}{\xi} K_{\mu+1}(\xi) K_{\mu}(\xi)\right]
$$

Thus the contribution to the cross-section from $b^{\prime} \leq b<\infty$ will be

$$
\sigma=\pi q^{2} b^{\prime 2} \frac{\xi^{2}}{2}\left[\left(K_{\mu+1}(\xi)\right)^{2}-\left(K_{\mu}(\xi)\right)^{2}-\frac{2 \mu}{\xi} K_{\mu+1}(\xi) K_{\mu}(\xi)\right]
$$

where

$$
\xi \equiv \frac{b^{\prime} \omega_{\text {on-shell }}}{\gamma v}
$$


and

$$
q^{2} \equiv \frac{\text { Born probability at } \mathrm{b}^{\prime}}{\left(K_{\mu}(\xi)\right)^{2}}
$$

In a situation such as that shown in Figure 8, where the Born formula does not give an adequate approximation to the exact result even at large $b$, it is necessary to do a numerical integration of $(2.9)$ over the entire $b$ range.

\subsection{Comparison of cross sections}

We use the term "exact" to refer to calculations in which the interaction matrix elements are evaluated without approximation, and full account is taken of coupling between 0-, 1-, and 2-phonon states. The LWLA calculations also include full coupling between 0-, 1-, and 2- phonon states, but the interaction matrix elements are evaluated approximately (Equation 2.12, and Section 4.3). The Born approximation uses the correct on-shell interaction matrix elements, but assumes that the transition to any final state is accomplished in a direct, single- step process. Of course, our "exact" calculation is not really exact, since it leaves out the effect of coupling with states of $3,4, \ldots$ phonons. For this reason, we have chosen to restrict our attention to bombarding energies of $\leq 10 \mathrm{~A} \mathrm{GeV}$, with the expectation that below this energy a description involving only $0-, 1-$, and 2-phonons will be adequate. This is probably a fairly good approximation for the excitation cross-sections of the 1-phonon states. It is difficult to assess the effect of our $\geq 3$-phonon truncation on the calculation of the 2-phonon cross-sections. This will be investigated in a future publication.

\subsubsection{The Born approximation}

A striking disagreement between the predictions of the Born approximation and the exact calculation is displayed in Figure 9. At bombarding energy below about $2 \mathrm{~A} \mathrm{GeV}$, the Born approximation and exact calculation yield nearly the same cross-section for population of the 1-phonon $M=0$ state. However, at higher bombarding energy, the Born approximation cross-section becomes vanishingly small, whereas the exact calculation predicts appreciable cross-section. It must be recalled that the Born approximation involves only the on- shell Fourier component of the interaction, $V_{\beta \alpha}\left(\omega=\frac{E_{\beta}-E_{\alpha}}{\hbar}\right)$. It was shown in Reference [25] that $\Delta M=0$ on-shell interaction matrix elements vanish at high bombarding energy in proportion to $1 / \gamma^{2}$, as a result of cancellation between the contributions of the scalar and vector potentials. This accounts for the strong decrease at high bombarding energy of the Born $\Delta M=0$ cross-section. However, off-shell interaction matrix elements,

$V_{\beta \alpha}\left(\omega \neq \frac{E_{\beta}-E_{\alpha}}{\hbar}\right)$, do not vanish at high $\gamma$; rather they diverge in proportion to $\log \gamma$. In the exact calculation, off-shell interaction matrix elements contribute to the population of the one-phonon $M=0$ state, as do multistep processes. Thus the strong Born approximation selection rule against population of the 1-phonon $M=0$ state is not exhibited by the exact calculation.

On the other hand, Figure 10 shows that the Born approximation and exact calculations are in fairly good agreement for the population of the 1-phonon $|M|=1$ state over the entire energy range. This suggests that most of the population of this state occurs in a 
single-step process. The fact that the exact calculation predicts a slightly smaller crosssection can be interpreted as a result of the loss of flux from the $|M|=1$ state associated with the coupling to other available states.

Figure 11 shows the cross-section for the total population of the 1- phonon level, including both the $M=0$ and the $|M|=1$ states. It is seen that as a result of the Born approximation underprediction for the $M=0$ state, and over-prediction for the $|M|=1$ state, there is fairly good agreement for the total 1-phonon cross-section over the entire energy range. Thus measurements of the total 1-phonon cross-section cannot distinguish between the predictions of the Born approximation and exact calculations, and our exact calculation has little to contribute to the many analyses of total 1-phonon cross-sections performed with the Born approximation. However, if the separate $M=0$ and $|M|=1$ cross-sections could be measured, then a clear choice could be made between the predictions of the Born approximation and the exact calculation.

Figures 12 through 16 show our results for the 2-phonon states. In all cases, the cross-section predicted by the exact calculation far exceeds the predictions of the Born approximation. The natural explanation is that these states are predominantly excited by multi-step processes.

\subsubsection{Comparison with the harmonic vibrator model}

Some analyses of multiphonon excitations have used the harmonic vibrator model, in which it is assumed that the total effect of the interaction on the target protons can be imitated by an operator that is linear in the components of the collective variable $\mathbf{R}_{\mathrm{pn}}$. This has the consequence that multi-phonon states are populated by a series of on-shell transitions, and thus the fact that the $\Delta M=0$ on-shell transition matrix element becomes small at high bombarding energy implies that the $\Delta M=0$ matrix elements will play little role in multiphonon excitation. It was shown in Reference [2] that the ratios of the crosssections for the 2-phonon states (020), (021), (022), (100) are predicted by this model to be $1 / 0 / 3 / 2$. The absence of population of the state $(021)$ is due to the fact that this state consists of phonons with $m=0$ and $m=1$, and the $m=0$ phonon is inaccessible. The predictions of the exact calculation, as shown in Figures 12 through 16, are quite different. At a bombarding energy of $10 \mathrm{~A} \mathrm{GeV}$, the ratios are approximately $4.1 / .3 / .1 / 1.6$. The important role of the $\Delta M=0$ matrix elements in these numbers is evident from the bombarding-energy dependence shown in Figures 12 through 16. For example, of these four states, only (022) shows a cross-section that decreases with bombarding energy, and this is the state which could be expected to be least affected by $\Delta M=0$ matrix elements. Thus, our microscopic treatment of the interaction, which follows the effect of the external pulse on each proton, gives quite different results from assumption that the interaction is linear in the collective variables. However, it must be borne in mind that the error we make by truncating our phonon space at two phonons may introduce significant errors into our 2-phonon predictions. This requires further investigation.

\subsubsection{The long-wavelength approximation (LWLA)}

It is seen in the cross-section comparisons, Figures 9 through 14, that at high bombarding energy the LWLA prediction exceeds the result of exact calculation, except for the 1- 
phonon $|M|=1$ state and the 2- phonon $|M|=2$ state. The common characteristic of these two states is that they involve only oscillation in the transverse $(\hat{x}, \hat{y})$ directions, whereas all the other final states involve oscillation in the $\hat{z}$ direction. We have already noted in connection with Figures 4 and 6 that the excitation of the $\hat{z}$ oscillation takes place rapidly in the vicinity of $t=0$. The LWLA matrix elements are more strongly peaked in the vicinity of $t=0$ (see Figures $1 \mathrm{~b}$ and $2 \mathrm{~b}$ ) due to absence of LWLA dynamic damping. Thus it is not surprising that the LWLA gives enhanced predictions for states involving $\hat{z}$ excitation. On the other hand, the excitation of the $\hat{x}, \hat{y}$ oscillation is a more gradual process, covering a larger $t$-range, and so is more sensitive to the time region in which the exact matrix elements are greater than the LWLA matrix elements (Figures 1b, 2b).

\section{Conclusions}

Although we have used a somewhat schematic representation of the GDR nuclear states, we can draw some conclusions about the extent to which the LWLA and Born approximation reproduce the results of our exact calculations. These conclusions may also apply to calculations that use more realistic nuclear states.

It is apparent from Figures 9 through 16 that at bombarding energies below about $3 \mathrm{~A} \mathrm{GeV}$, the LWLA and the exact calculations predict essentially the same cross-sections, state by state. This observation supports the validity of several RCE investigations that have used the LWLA [5, 6. It is shown in Figure 12 that the agreement between the LWLA and exact calculations of the total 1-phonon cross-section extends up to about $5 \mathrm{~A} \mathrm{GeV}$. Above $5 \mathrm{~A} \mathrm{GeV}$, however, there are significant deviations between the LWLA and exact cross-sections. Thus at higher bombarding enrgyies, the LWLA ceases to be a reliable approximation, especially if it is important to know the cross-sections for population of individual $M$-states.

Not surprisingly, the Born approximation is unable to predict the cross- section for population of 2-phonon states. Its performance with respect to the 1-phonon states is analagous to that of the LWLA. At bombarding energies below $2 \mathrm{~A} \mathrm{GeV}$, it does well with both $M=0$ and $|M|=1$ states. At higher energies, it underpredicts the $M=0$ crosssection (Figure 9), and over-predicts the $|M|=1$ cross-section (Figure 10). The net result is good agreement with the exact prediction for the total 1-phonon cross-section up to about $8 \mathrm{~A} \mathrm{GeV}$ (Figure 11).

\section{Acknowledgements}

The authors wish to thank A. Vitturi for his suggestion that they investigate the use of numerical Fourier transforms to calculate the time-dependent interaction matrix elements. One of us (B.F.B) acknowledges financial support from the INFN.

\section{Appendices}

\section{A: Brink's representation of the GDR phonon states}

We first construct the states of the GDR which are automatically contained in the independent particle harmonic oscillator shell model. For simplicity, we restrict our attention 
to $N=Z$ closed-shell nuclei. We start by locating the $Z$ protons and the $Z$ neutrons relative to the fixed harmonic oscillator origin by $\mathbf{p}_{1}, \cdots, \mathbf{p}_{Z}, \mathbf{n}_{1}, \cdots, \mathbf{n}_{Z}$. To construct GDR states, we introduce new position variables

$$
\begin{aligned}
\mathbf{R} & =\frac{\mathbf{p}_{1}+\cdots+\mathbf{p}_{Z}+\mathbf{n}_{1}+\cdots+\mathbf{n}_{Z}}{2 Z} \\
\mathbf{R}_{\mathrm{pn}} & =\left(\frac{\mathbf{p}_{1}+\cdots+\mathbf{p}_{Z}}{Z}\right)-\left(\frac{\mathbf{n}_{1}+\cdots+\mathbf{n}_{Z}}{Z}\right)
\end{aligned}
$$

and relative variables $\pi_{1}, \cdots, \pi_{Z-1}, \nu_{1}, \cdots, \nu_{Z-1}$. The $\pi_{1}, \cdots, \pi_{Z-1}$ specify the location of the $Z$ protons relative to their mass center.

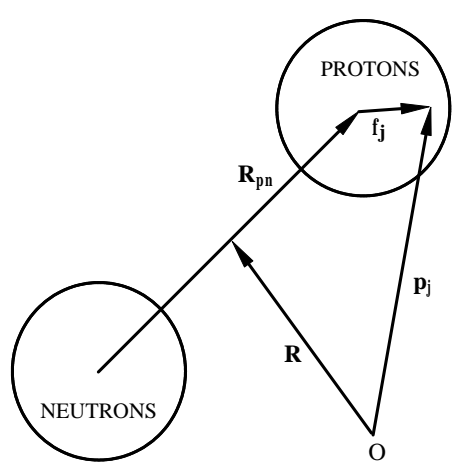

Figure A1. $\mathbf{R}_{\mathrm{pn}}$ locates the position of the proton mass-center relative to the neutron mass center, whereas $\mathbf{R}$ locates the total mass center. The $j^{\text {th }}$ proton is located relative to the proton mass center by $\mathbf{f}_{j}$.

It is clear from Fig.(A1) that the $j$ th proton is located relative to the origin by

$$
\mathbf{p}_{j}=\mathbf{R}+\frac{1}{2} \mathbf{R}_{\mathrm{pn}}+\mathbf{f}_{j}\left(\boldsymbol{\pi}_{1}, \cdots, \boldsymbol{\pi}_{Z-1}\right)
$$

We will not have to specify the $\boldsymbol{\pi}_{1}, \cdots, \boldsymbol{\pi}_{Z-1}, \boldsymbol{\nu}_{1}, \cdots, \boldsymbol{\nu}_{Z-1}$ or the $\mathbf{f}_{j}$ any further; we will only need the $\mathbf{R}$ and the $\mathbf{R}_{\mathrm{pn}}$ dependence of $\mathbf{p}_{j}$.

If the independent-particle harmonic oscillator Hamiltonian is expressed in terms of these new variables, it separates as follows:

$$
\begin{aligned}
H & =\left(-\frac{\hbar^{2}}{2 \cdot(2 Z m)} \nabla_{\mathbf{R}}^{2}+\frac{2 Z m \omega_{\mathrm{sm}}^{2}}{2} \mathrm{R}^{2}\right)+\left(-\frac{\hbar^{2}}{2\left(\frac{Z}{2} m\right)} \nabla_{\mathbf{R}_{\mathrm{pn}}}^{2}+\frac{\frac{Z}{2} m \omega_{\mathrm{sm}}^{2}}{2} \mathrm{R}_{\mathrm{pn}}^{2}\right) \\
& +H^{\prime}\left(\boldsymbol{\pi}_{\boldsymbol{1}}, \cdots, \boldsymbol{\pi}_{\boldsymbol{Z}-\mathbf{1}}, \boldsymbol{\nu}_{\mathbf{1}}, \cdots, \boldsymbol{\nu}_{\boldsymbol{Z}-\mathbf{1}}\right) .
\end{aligned}
$$


Here $m$ is the nucleon mass and $\omega_{\text {sm }}$ is the single- nucleon oscillator frequency. Corresponding to this separability, we can write our harmonic oscillator eigenstates in the form

$$
\Psi_{\alpha}=\Psi_{M_{1}}^{N_{1}, L_{1}}(\mathbf{R}) \Psi_{M_{2}}^{N_{2}, L_{2}}\left(\mathbf{R}_{\mathrm{pn}}\right) \times \chi\left(\boldsymbol{\pi}_{1}, \cdots, \boldsymbol{\pi}_{Z-1}, \quad \boldsymbol{\nu}_{\mathbf{1}}, \cdots, \boldsymbol{\nu}_{\mathbf{Z}-\mathbf{1}}\right) .
$$

The spin degrees of freedom are incorporated in $\chi$, in such a way as to produce a state which is antisymmetric with respect to the exchange of any two protons or any two neutrons. Note that changing $N_{1}, L_{1}, M_{1}$ or $N_{2}, L_{2}, M_{2}$ has no effect on the antisymmetry, since the variables $\mathbf{R}$ and $\mathbf{R}_{\mathrm{pn}}$ are symmetric with respect to the exchange of any two protons or any two neutrons.

It is clear that the state of lowest energy, consistent with the Pauli principle, will have zero quanta in the variables $\mathbf{R}$ and $\mathbf{R}_{\mathrm{pn}}$ :

$$
\Psi_{\text {ground-state }}=\Psi_{0}^{0,0}(\mathbf{R}) \Psi_{0}^{0,0}\left(\mathbf{R}_{\mathrm{pn}}\right) \times \chi_{0}\left(\boldsymbol{\pi}_{1}, \cdots, \boldsymbol{\pi}_{Z-1}, \nu_{1}, \cdots, \nu_{Z-1}\right) .
$$

Now let us consider a series of states of the form

$$
\Psi_{M}^{N, L}=\Psi_{0}^{0,0}(\mathbf{R}) \Psi_{M}^{N, L}\left(\mathbf{R}_{\mathrm{pn}}\right) \times \chi_{0}\left(\boldsymbol{\pi}_{1}, \cdots, \boldsymbol{\pi}_{Z-1}, \boldsymbol{\nu}_{\mathbf{1}}, \cdots, \boldsymbol{\nu}_{\boldsymbol{Z}-\mathbf{1}}\right) .
$$

These states all have no quanta of center-of-mass motion. The relative motions of the protons to each other, and of the neutrons to each other, are exactly the same as they are in the shell-model ground state. The only feature distinguishing the states (A6) is the motion of the proton mass-center relative to the neutron mass-center. Brink [29] identified these states as belonging to the GDR, because this collective oscillation of the protons relative to the neutrons was the original interpretation given by Goldhaber and Teller 27] to the phenomenon now known as the GDR.

It can be seen from (A3) that the energy associated with the $\mathbf{R}_{\mathrm{pn}}$ quanta is $\hbar \omega_{\mathrm{sm}}$, the same as the energy associated with the individual oscillator quanta. It was suggested by Wilkinson [28, and proved by Brown and Bolsterli [31, that the residual interaction between the nucleons will increase the GDR phonon energy.

The harmonic oscillator radial functions associated with the individual nucleons were specified in (4.6). The same radial functions are used for the GDR states $\Psi_{M}^{N, L}\left(\mathbf{R}_{\mathrm{pn}}\right)$, except that $m$ is replaced by $Z m / 2$, which is the reduced mass for the relative motion of the proton and neutron spheres.

Electromagnetic properties of states are normally discussed in the long-wavelength approximation, in which the electric dipole operator has the form

$$
\mathcal{M}=\sqrt{\frac{3}{4 \pi}} \sum_{j=1}^{Z} \mathbf{p}_{\mathrm{j}}
$$

Equation (A.2) can be used to express this operator in terms of $\mathbf{R}, \mathbf{R}_{\mathrm{pn}}, \boldsymbol{\pi}_{j}$. The only part of this operator that has matrix elements between states of the GDR band (A.6) is

$$
\mathcal{M}=\sqrt{\frac{3}{4 \pi}} \frac{Z}{2} \mathbf{R}_{\mathrm{pn}}
$$

which can only connect states which differ by one GDR phonon. Thus the E1 transition between the ground state and the 1-phonon Brink level exhausts the E1 sum rule. 


\section{B: Contribution of the scalar potential}

It is shown in [25] that if the nuclear states $\phi_{\alpha}, \phi_{\beta}$ are defined with "time-reversal" phases,

$$
\left(\phi_{M_{\alpha}}^{I_{\alpha}}\right)^{*}=(-1)^{I_{\alpha}-M_{\alpha}} \phi_{M_{-\alpha}}^{I_{\alpha}}
$$

then the $V_{\beta \alpha}(\omega)$ will be purely real. We will use this phase convention.

The charge density $\rho_{\beta \alpha}\left(\mathbf{r}^{\prime}\right)$ is defined in terms of the nuclear eigenstates $\phi_{\beta}, \phi_{\alpha}$ by

$$
\rho_{\beta \alpha}\left(\mathbf{r}^{\prime}\right)=e \int d^{3} p_{1} \ldots d^{3} n_{Z} \sum_{j=1}^{Z} \delta\left(\mathbf{r}^{\prime}-\mathbf{p}_{j}\right) \phi_{\beta}^{*} \phi_{\alpha}
$$

The sum extends over the protons only. By using (B2) and (4.3) in (4.1), we see that the scalar potential contribution to $V_{\beta \alpha}(\omega)$ is

$$
\int d^{3} r^{\prime} \varphi_{C}^{\mathrm{ret}}\left(\mathbf{r}^{\prime}, \omega\right) \rho_{\beta \alpha}\left(\mathbf{r}^{\prime}\right)=\sum_{\lambda \mu} C_{\lambda, \mu}(\omega) K_{\mu}\left(\frac{|\omega| b}{v \gamma}\right) \int d^{3} r^{\prime} \rho_{\beta \alpha}\left(\mathbf{r}^{\prime}\right) j_{\lambda}\left(\frac{|\omega|}{c} r^{\prime}\right) Y_{\mu}^{\lambda}\left(\hat{r}^{\prime}\right)
$$

The integral required here also occurs in the Fourier transform of $\rho_{\beta \alpha}\left(\mathbf{r}^{\prime}\right)$ :

$$
\rho_{\beta \alpha}(\mathbf{k}) \equiv \int e^{i \mathbf{k} \cdot \mathbf{r}^{\prime}} \rho_{\beta \alpha}\left(\mathbf{r}^{\prime}\right) d^{3} r^{\prime}=4 \pi \sum_{\lambda \mu} i^{\lambda} Y_{\mu}^{\lambda *}(\hat{\mathbf{k}}) \int d^{3} r^{\prime} \rho_{\beta \alpha}\left(\mathbf{r}^{\prime}\right) j_{\lambda}\left(k r^{\prime}\right) Y_{\mu}^{\lambda}\left(\hat{r}^{\prime}\right)
$$

if we set $k=|\omega| / c$. Thus we turn our attention to the evaluation of $\rho_{\beta \alpha}(\mathbf{k})$ :

$$
\begin{gathered}
\rho_{\beta \alpha}(\mathbf{k}) \equiv \int e^{i \mathbf{k} \cdot \mathbf{r}^{\prime}} \rho_{\beta \alpha}\left(\mathbf{r}^{\prime}\right) d^{3} r^{\prime}=\int d^{3} r^{\prime} e^{i \mathbf{k} \cdot \mathbf{r}^{\prime}}<\phi_{\beta}\left|e \sum_{j=1}^{Z} \delta\left(\mathbf{r}^{\prime}-\mathbf{p}_{j}\right)\right| \phi_{\alpha}> \\
=<\phi_{\beta}\left|e \sum_{j=1}^{Z} e^{i \mathbf{k} \cdot \mathbf{p}^{\prime}{ }_{j}}\right| \phi_{\alpha}>.
\end{gathered}
$$

We now choose states in the GDR band (A6) for the $\phi_{\beta}$ and $\phi_{\alpha}$, and use (A2) to express $\mathbf{p}_{j}$ in terms of the new variables:

$$
\begin{aligned}
\rho_{\beta \alpha}(\mathbf{k}) & =e \int d^{3} R\left|\Psi_{0}^{0,0}\right|^{2} \times \int d^{3} R_{\mathrm{pn}} \Psi_{M_{\beta}}^{* N_{\beta}, L_{\beta}}\left(\mathbf{R}_{\mathrm{pn}}\right) \Psi_{M_{\alpha}}^{N_{\alpha}, L_{\alpha}}\left(\mathbf{R}_{\mathrm{pn}}\right) \\
& \times \int d^{3} \boldsymbol{\pi}_{1}, \cdots, \boldsymbol{d}^{3} \boldsymbol{\nu}_{Z-1}\left|\chi_{0}\left(\boldsymbol{\pi}_{1}, \cdots, \boldsymbol{\pi}_{Z-1}, \boldsymbol{\nu}_{1}, \cdots, \boldsymbol{\nu}_{Z-1}\right)\right|^{2} \\
& \times \sum_{j=1}^{Z} e^{i \mathbf{k} \cdot\left(\mathbf{R}+\frac{1}{2} \mathbf{R}_{p n}+\mathbf{f}_{j}\left(\boldsymbol{\pi}_{1}, \cdots, \boldsymbol{\pi}_{Z-1}, \quad \boldsymbol{\nu}_{1}, \cdots, \boldsymbol{\nu}_{Z-1}\right)\right.} \\
& =Q \int d^{3} R_{\mathrm{pn}} \Psi_{M_{\beta}}^{* N_{\beta}, L_{\beta}}\left(\mathbf{R}_{\mathrm{pn}}\right) e^{i \frac{\mathbf{k}}{2} \cdot \mathbf{R}_{\mathrm{pn}}} \Psi_{M_{\alpha}}^{N_{\alpha}, L_{\alpha}}\left(\mathbf{R}_{\mathrm{pn}}\right)
\end{aligned}
$$

with

$$
\begin{aligned}
Q & \equiv e \int d^{3} R\left|\Psi_{0}^{0,0}\right|^{2} e^{i \mathbf{k} \cdot \mathbf{R}} \times \int d^{3} \boldsymbol{\pi}_{1}, \cdots, \boldsymbol{d}^{3} \boldsymbol{\nu}_{Z-1}\left|\chi_{0}\left(\boldsymbol{\pi}_{1}, \cdots, \boldsymbol{\pi}_{Z-1}, \boldsymbol{\nu}_{1}, \cdots, \boldsymbol{\nu}_{Z-1}\right)\right|^{2} \\
& \times \sum_{j=1}^{Z} e^{i \mathbf{k} \cdot \mathbf{f}_{j}\left(\boldsymbol{\pi}_{1}, \cdots, \boldsymbol{\pi}_{Z-1}, \quad \boldsymbol{\nu}_{1}, \cdots, \boldsymbol{\nu}_{Z-1}\right)}
\end{aligned}
$$


Note that the factor $Q$ is independent of the GDR quantum numbers. This is a consequence of our assumption that the proton and neutron spheres remain unexcited during the $\mathbf{R}_{\mathrm{pn}}$ oscillation. Thus we can calculate $Q$ by considering the ground-state proton density

$\rho_{00}(\mathbf{k})=e \int d^{3} r^{\prime} e^{i \mathbf{k} \cdot \mathbf{r}^{\prime}} \frac{1}{4 \pi} \sum_{n^{\prime}, \ell} 2(2 \ell+1) u_{n^{\prime}, \ell}^{2}\left(r^{\prime}\right)=e \sum_{n^{\prime}, \ell} 2(2 \ell+1) \int_{0}^{\infty} u_{n^{\prime}, \ell}^{2}\left(r^{\prime}\right) j_{0}\left(k r^{\prime}\right) r^{\prime} 2 d r^{\prime}$

The $n^{\prime}, \ell$ sum extends over the single-particle levels occupied in the ground state (for example: $00,01,02,10$, for ${ }^{40} \mathrm{Ca}$ ). Using $(\mathrm{B} 6)$ to evaluate $\rho_{00}(\mathbf{k})$ yields

$$
\rho_{00}(\mathbf{k})=e^{-\frac{k^{2}}{8 Z \nu}} \times Q
$$

so that

$$
Q=e^{\frac{k^{2}}{8 Z \nu}} \times e \sum_{n, \ell} 2(2 \ell+1) \int_{0}^{\infty} u_{n, \ell}^{2}\left(r^{\prime}\right) j_{0}\left(k r^{\prime}\right) r^{\prime 2} d r^{\prime} .
$$

To evaluate the $\mathbf{R}_{\mathrm{pn}}$ integral in (B6), we write

$$
\begin{gathered}
\Psi_{M_{\beta}}^{* N_{\beta}, L_{\beta}}\left(\mathbf{R}_{\mathrm{pn}}\right) \Psi_{M_{\alpha}}^{N_{\alpha}, L_{\alpha}}\left(\mathbf{R}_{\mathrm{pn}}\right)=(-1)^{L_{\beta}-M_{\beta}} \Psi_{-M_{\beta}}^{N_{\beta}, L_{\beta}}\left(\mathbf{R}_{\mathrm{pn}}\right) \Psi_{M_{\alpha}}^{N_{\alpha}, L_{\alpha}}\left(\mathbf{R}_{\mathrm{pn}}\right) \\
=(-1)^{L_{\beta}-M_{\beta}} \sum_{L}\left(L_{\beta} L_{\alpha}-M_{\beta} M_{\alpha} \mid L M_{\alpha}-M_{\beta}\right) \times\left[\Psi^{N_{\beta}, L_{\beta}}\left(\mathbf{R}_{\mathrm{pn}}\right) \Psi^{N_{\alpha}, L_{\alpha}}\left(\mathbf{R}_{\mathrm{pn}}\right)\right]_{M_{\alpha}-M_{\beta}}^{L}
\end{gathered}
$$

We then use the Talmi-Moshinsky coefficients, defined by

$$
\begin{aligned}
{\left[\psi^{n_{1} \ell_{1}}\left(\mathbf{r}_{1}\right) \psi^{n_{2} \ell_{2}}\left(\mathbf{r}_{2}\right)\right]_{M}^{L}=} & \sum_{n, \lambda, N, \Lambda} i^{\ell_{1}+\ell_{2}-\lambda-\Lambda}\left(n_{1} \ell_{1}, n_{2} \ell_{2} \mid n \lambda, N \Lambda\right)_{L} \\
& {\left[\psi^{n \lambda}\left(\frac{\mathbf{r}_{1}-\mathbf{r}_{2}}{\sqrt{2}}\right) \psi^{N \Lambda}\left(\frac{\mathbf{r}_{1}+\mathbf{r}_{2}}{\sqrt{2}}\right)\right]_{M}^{L} }
\end{aligned}
$$

with $\mathbf{r}_{1}=\mathbf{r}_{2}=\mathbf{R}_{\mathrm{pn}}$, to write

$$
\begin{aligned}
& {\left[\Psi^{N_{\beta}, L_{\beta}}\left(\mathbf{R}_{\mathrm{pn}}\right) \Psi^{N_{\alpha}, L_{\alpha}}\left(\mathbf{R}_{\mathrm{pn}}\right)\right]_{M_{\alpha}-M_{\beta}}^{L} } \\
= & \sum_{n, \lambda, N, \Lambda} i^{L_{\alpha}+L_{\beta}-\lambda-\Lambda}\left(N_{\beta} L_{\beta}, N_{\alpha} L_{\alpha} \mid n \lambda, N \Lambda\right)_{L}\left[\Psi^{n \lambda}(0) \psi^{N \Lambda}\left(\sqrt{2} \mathbf{R}_{\mathrm{pn}}\right)\right]_{M_{\alpha}-M_{\beta}}^{L} \\
= & i^{\ell_{1}+\ell_{2}-L}\left(\frac{Z \nu}{2 \pi}\right)^{3 / 4} \sum_{n, N}\left(N_{\beta} L_{\beta}, N_{\alpha} L_{\alpha} \mid n 0, N L\right)_{L} \times \sqrt{\frac{(2 n+1) ! !}{2 n ! !}} \Psi_{M_{\alpha}-M_{\beta}}^{N L}\left(\sqrt{2} \mathbf{R}_{\mathrm{pn}}\right)
\end{aligned}
$$

The extra factor $i^{\ell_{1}+\ell_{2}-\lambda-\Lambda}$ is due to our use of time-reversal phases. The $n, N$ sum in (B13) is restricted by the quanta-preserving condition $2(n+N)+L=2\left(N_{\beta}+N_{\alpha}\right)+L_{\beta}+L_{\alpha}$, which in turn requires that $L_{\beta}+L_{\alpha}-L$ be even.

To Fourier transform $\Psi_{M_{\alpha}-M_{\beta}}^{N L}\left(\sqrt{2} \mathbf{R}_{\mathrm{pn}}\right)$ we use the general harmonic oscillator result

$$
\frac{1}{(2 \pi)^{3 / 2}} \int_{-\infty}^{\infty} e^{i \mathbf{k} \cdot \mathbf{r}} \psi_{m}^{n \ell}(\mathbf{r}) d^{3} r=i^{2 n+\ell} \tilde{\psi}_{m}^{n \ell}(\mathbf{k})
$$


where $\tilde{\psi}_{m}^{n \ell}(\mathbf{k})$ has a size parameter $\tilde{\nu}$ which is the reciprocal of the size parameter of $\psi_{\mu}^{n \ell}(\mathbf{r})$. Then

$$
\begin{gathered}
\int_{-\infty}^{\infty} e^{i \frac{\mathbf{k}}{2} \cdot \mathbf{R}_{\mathrm{pn}}} \Psi_{M_{\alpha}-M_{\beta}}^{N L}\left(\sqrt{2} \mathbf{R}_{\mathrm{pn}}\right) d^{3} \mathbf{R}_{\mathrm{pn}}=i^{2 N+L} \pi^{3 / 2} \tilde{\Psi}_{M_{\alpha}-M_{\beta}}^{N L}\left(\frac{\mathbf{k}}{2 \sqrt{2}}\right) \\
=(-1)^{N+L} \pi^{3 / 2} Y_{M_{\alpha}-M_{\beta}}^{L}(\hat{\mathbf{k}}) \tilde{u}_{N L}\left(\frac{k}{2 \sqrt{2}}\right) .
\end{gathered}
$$

By combining (B6), (B8), (B13) and (B14), we get

$$
\begin{aligned}
\rho_{\beta \alpha}(\mathbf{k})= & (-1)^{M_{\beta}} i^{L_{\alpha}-L_{\beta}}\left(\frac{Z \nu \pi}{2}\right)^{3 / 4} e^{\frac{k^{2}}{8 Z \nu}} \sum_{n^{\prime}, \ell} 2(2 \ell+1) \int_{0}^{\infty} j_{0}\left(k r^{\prime}\right) u_{n^{\prime}, \ell}^{2}\left(r^{\prime}\right) r^{\prime 2} d r^{\prime} \\
& \times \sum_{n, N, L} i^{2 N+L}\left(L_{\beta} L_{\alpha}-M_{\beta} M_{\alpha} \mid L M_{\alpha}-M_{\beta}\right) \\
& \times\left(N_{\beta} L_{\beta}, N_{\alpha} L_{\alpha} \mid n 0, N L\right)_{L} \times \sqrt{\frac{(2 n+1) ! !}{2 n ! !}} \tilde{u}_{N L}\left(\frac{k}{2 \sqrt{2}}\right) Y_{M_{\alpha}-M_{\beta}}^{L}(\hat{\mathbf{k}})
\end{aligned}
$$

Finally, comparison with (B3) and (B4) shows that

$$
\begin{aligned}
& \int d^{3} r^{\prime} \varphi_{C}^{\mathrm{ret}}\left(\mathbf{r}^{\prime}, \omega\right) \rho_{\beta \alpha}\left(\mathbf{r}^{\prime}\right)=\frac{(-1)^{M_{\alpha}} i^{L_{\alpha}-L_{\beta}}}{4 \pi}\left(\frac{Z \nu \pi}{2}\right)^{3 / 4} e^{\frac{(\mid \omega / c)^{2}}{8 Z \nu}} K_{\mu}\left(\frac{(}{|\omega| b} v \gamma\right) \\
& \times \sum_{n, \ell} 2(2 \ell+1) \int_{0}^{\infty} j_{0}\left(\frac{|\omega|}{c} r^{\prime}\right) u_{n, \ell}^{2}\left(r^{\prime}\right) r^{\prime 2} d r^{\prime} \sum_{L} C_{L, M_{\beta}-M_{\alpha}}\left(L_{\beta} L_{\alpha}-M_{\beta} M_{\alpha} \mid L M_{\alpha}-M_{\beta}\right) \\
& \times \sum_{n, N}(-1)^{N}\left(N_{\beta} L_{\beta}, N_{\alpha} L_{\alpha} \mid n 0, N L\right)_{L} \sqrt{\frac{(2 n+1) ! !}{2 n ! !}} \tilde{u}_{N L}\left(\frac{\frac{|\omega|}{c}}{2 \sqrt{2}}\right),
\end{aligned}
$$

which gives us an explicit expression for the contribution of the scalar potential to $V_{\beta \alpha}(\omega)$. This expression is summarized in (4.5)

\section{C: Contribution of the vector potential}

The analysis is similar to the one presented in Appendix B for the scalar potential. We need

$$
\begin{aligned}
-\frac{1}{c} \int d^{3} r^{\prime} \mathbf{j}_{\beta \alpha}\left(\mathbf{r}^{\prime}\right) \cdot \mathbf{A}_{C}^{\mathrm{ret}}\left(\mathbf{r}^{\prime}, \omega\right) & =-\frac{v}{c^{2}} \int d^{3} r^{\prime}\left[\mathbf{j}_{\beta \alpha}\left(\mathbf{r}^{\prime}\right)\right]_{z} \varphi_{C}^{\mathrm{ret}}\left(\mathbf{r}^{\prime}, \omega\right) \\
& =-\frac{v}{c^{2}} \sum_{\lambda, \mu} C_{\lambda, \mu} \int d^{3} r j_{\lambda}\left(k r^{\prime}\right) Y_{\mu}^{\lambda}\left(\hat{\mathbf{r}^{\prime}}\right)\left[\mathbf{j}_{\beta \alpha}\left(\mathbf{r}^{\prime}\right)\right]_{z}
\end{aligned}
$$

To obtain these integrals we study the Fourier transform of $\left[\mathbf{j}_{\beta \alpha}\left(\mathbf{r}^{\prime}\right)\right]_{z}$ :

$$
\begin{aligned}
{\left[\mathbf{j}_{\beta \alpha}(\mathbf{k})\right]_{z} } & \equiv \int e^{i \mathbf{k} \cdot \mathbf{r}^{\prime}}\left[\mathbf{j}_{\beta \alpha}\left(\mathbf{r}^{\prime}\right)\right]_{z} d^{3} r^{\prime}=4 \pi \sum_{\lambda, \mu} i^{\lambda} Y_{\mu}^{* \lambda}(\hat{\mathbf{k}}) \int d^{3} r j_{\lambda}\left(k r^{\prime}\right)\left[\mathbf{j}_{\beta \alpha}\left(\mathbf{r}^{\prime}\right)\right]_{z} \\
& =\int d^{3} r e^{i \mathbf{k} \cdot \mathbf{r}^{\prime}} \int d^{3} p_{1} \cdots d^{3} n_{Z} \frac{e \hbar}{2 m i} \sum_{j=1}^{Z} \delta\left(\mathbf{r}^{\prime}-\mathbf{p}_{j}\right)\left(\phi_{\beta}^{*} \frac{\partial}{\partial p_{j, z}} \phi_{\alpha}-\phi_{\alpha} \frac{\partial}{\partial p_{j, z}} \phi_{\beta}^{*}\right) \\
& =\int d^{3} p_{1}, \cdots d^{3} n_{Z} \sum_{j=1}^{Z} e^{i \mathbf{k} \cdot \mathbf{p}_{\mathbf{j}}} \times\left(\phi_{\beta}^{*} \frac{\partial}{\partial p_{j, z}} \phi_{\alpha}-\phi_{\alpha} \frac{\partial}{\partial p_{j, z}} \phi_{\beta}^{*}\right)
\end{aligned}
$$


We now take $\phi_{\beta}, \phi_{\alpha}$ to be states in the GDR band (A6), and transform to the variables $\mathbf{R}, \mathbf{R}_{\mathrm{pn}}, \boldsymbol{\pi}_{1}, \cdots, \boldsymbol{\pi}_{Z-1}, \boldsymbol{\nu}_{1}, \cdots, \boldsymbol{\nu}_{Z-1}$. To do this we use (A2) to obtain

$$
\frac{\partial}{\partial p_{j, z}}=\frac{1}{2 Z} \frac{\partial}{\partial \mathrm{R}_{z}}+\frac{1}{Z} \frac{\partial}{\partial \mathrm{R}_{\mathrm{pn}, z}}+\sum_{k=1 \rightarrow j-1 ; \mu=x, y, z}\left(\frac{\partial}{\partial p_{j, z}} \pi_{k, \mu}\right) \frac{\partial}{\partial \pi_{k, \mu}}
$$

Since the $\boldsymbol{\pi}_{k}$ involve only the relative positions of the $\mathbf{p}_{j}$, the quantities $\frac{\partial}{\partial p_{j, z}} \pi_{k, \mu}$ are independent of $\mathbf{R}$ and $\mathbf{R}_{\mathrm{pn}}$

Equation (C2) now takes the form

$$
\begin{gathered}
{\left[\mathbf{j}_{\beta \alpha}(\mathbf{k})\right]_{z}=\frac{e \hbar}{2(Z m i)} Q \times \int d^{3} R_{\mathrm{pn}} e^{\frac{\mathbf{k} \cdot \mathbf{R}_{\mathrm{pn}}}{2}}} \\
\times\left(\Psi_{M_{\beta}}^{* N_{\beta} L_{\beta}}\left(\mathbf{R}_{\mathrm{pn}}\right) \frac{\partial}{\partial R_{\mathrm{pn}, z}} \Psi_{M_{\alpha}}^{N_{\alpha} L_{\alpha}}\left(\mathbf{R}_{\mathrm{pn}}\right)-\Psi_{M_{\alpha}}^{N_{\alpha} L_{\alpha}} \frac{\partial}{\partial R_{\mathrm{pn}, z}} \Psi_{M_{\beta}}^{* N_{\beta} L_{\beta}}\left(\mathbf{R}_{\mathrm{pn}}\right)\right)
\end{gathered}
$$

where $Q$ is the quantity defined in (B7) and evaluated in (B10). Note that (C3) vanishes for diagonal matrix elements $\left(N_{\beta}=N_{\alpha}, L_{\beta}=L_{\alpha}, M_{\beta}=M_{\alpha}\right)$. The derivatives in (C3) can be expressed as linear combinations of harmonic oscillator wave functions using the relation

$$
\begin{gathered}
\frac{\partial}{\partial Z} \Psi_{m}^{n, \ell}(\mathbf{R})=\sqrt{\frac{\nu}{2}} \times \\
{\left[\sqrt{\frac{(\ell-m)(\ell+m)}{(2 \ell-1)(2 \ell+1)}}\left[\sqrt{2 n+2 \ell+1} \Psi_{m}^{n, \ell-1}(\mathbf{R})+\sqrt{2 n+2} \Psi_{m}^{n+1, \ell-1}(\mathbf{R})\right]\right.} \\
\left.-\sqrt{\frac{(\ell+1-m)(\ell+1+m)}{(2 \ell+1)(2 \ell+3)}}\left[\sqrt{2 n} \Psi_{m}^{n-1, \ell+1}(\mathbf{R})+\sqrt{2 n+2 \ell+3} \Psi_{m}^{n, \ell+1}(\mathbf{R})\right]\right]
\end{gathered}
$$

This converts (C3) into a linear combination of terms such as (B6), which can be evaluated exactly as was done in Appendix B.

\section{D: Symmetries}

Since the projectile moves in the $y-z$ plane, its scalar and vector potentials will be invariant under reflection across the $y-z$ plane. Therefore, only reflection-symmetric target states will be excited from the reflection-symmetric ground state. These are

$$
\Psi_{0}^{N L}, \quad \text { or } \quad \frac{1}{\sqrt{2}}\left(\Psi_{|M|}^{N L}+\Psi_{-|M|}^{N L}\right) .
$$

Thus if $M_{\alpha} \neq 0$ and $M_{\beta} \neq 0$, we must calculate

$$
\begin{gathered}
<\frac{1}{\sqrt{2}}\left(\Psi_{M_{\beta}}^{N_{\beta} L_{\beta}}+\Psi_{-M_{\beta}}^{N_{\beta} L_{\beta}}\right)|V(\omega)| \frac{1}{\sqrt{2}}\left(\Psi_{M_{\alpha}}^{N_{\alpha} L_{\alpha}}+\Psi_{-M_{\alpha}}^{N_{\alpha} L_{\alpha}}\right)> \\
=<\Psi_{\left|M_{\beta}\right|}^{N_{\beta} L_{\beta}}|V(\omega)| \Psi_{\left|M_{\alpha}\right|}^{N_{\alpha} L_{\alpha}}>+<\Psi_{\left|M_{\beta}\right|}^{N_{\beta} L_{\beta}}|V(\omega)| \Psi_{-\left|M_{\alpha}\right|}^{N_{\alpha} L_{\alpha}}>.
\end{gathered}
$$


If $M_{\beta} \neq 0$ and $M_{\alpha}=0$, we must calculate

$$
<\frac{1}{\sqrt{2}}\left(\Psi_{M_{\beta}}^{N_{\beta} L_{\beta}}+\Psi_{-M_{\beta}}^{N_{\beta} L_{\beta}}\right)|V(\omega)| \Psi_{0}^{N_{\alpha} L_{\alpha}}>=\sqrt{2}<\Psi_{\left|M_{\beta}\right|}^{N_{\beta} L_{\beta}}|V(\omega)| \Psi_{0}^{N_{\alpha} L_{\alpha}}>.
$$

It can be verified that Equation B3 of Reference [25]

$$
<\Psi_{M_{\beta}}^{N_{\beta} L_{\beta}}|V(\omega)| \Psi_{M_{\alpha}}^{N_{\alpha} L_{\alpha}}>=<\Psi_{M_{\alpha}}^{N_{\alpha} L_{\alpha}}|V(-\omega)| \Psi_{M_{\beta}}^{N_{\beta} L_{\beta}}>,
$$

is satisfied by the matrix elements discussed in Appendices B and C.

\section{References}

[1] A. Winther and K. Alder, Nucl. Phys. A319 518 (1979).

[2] C.A. Bertulani and G. Baur, Phys. Rep. 163299 (1988).

[3] H. Emling, Progr. Part. Nucl. Phys. 33792 (1994).

[4] Ph. Chomaz and N. Frascaria, Phys. Rep. 252, 275 (1995).

[5] C.A. Bertulani, F. Canto, M.S. Hussein and A.F.R. de Toledo Piza, Phys. Rev. C 53 334 (1996).

[6] E.G. Lanza, M.V. Andres, F. Catara, Ph. Chomaz, and, C. Volpe, Nucl. Phys. A613 445 (1997).

[7] T. Aumann, P.F. Bortignon and H. Emling, Annu. Rev. Nucl. Part. Sci. 48351 (1998).

[8] C.A. Bertulani and V.Yu. Ponomarev, Phys. Rep. 321139 (1999).

[9] A.J. Baltz, M.J. Rhoades-Brown and J. Weneser, Phys. Rev. E 544233 (1996).

[10] S. Datz et al., Phys. Rev. Lett. 793355 (1997).

[11] I.A. Pshenichnov et al., Phys. Rev. C 57, 1920 (1998).

[12] H.H. Heckman and P.J. Lindstrom, Phys. Rev. Lett. 3756 (1976).

[13] T. Aumann, C.A. Bertulani and K. Sümmerer, Phys. Rev. C 51 (1995)

[14] J.D. Jackson, Classical Electrodynamics (Wiley, New York, 1975).

[15] M.T. Mercier et al, Phys. Rev. C 331655 (1986).

[16] T. Aumann et al, Phys. Rev. C47 1728 (1993).

[17] J.Z. Gu and H.A Weidenmüller, Nucl. Phys. A690 382 (2001).

[18] V.Yu. Ponomarev et al, Phys. Rev. Lett. 721168 (1994). 
[19] V.Yu. Ponomarev, P.F. Bortignon, R.A. Broglia and V.V. Voronov, Nucl. Phys. A687 170c (2001).

[20] B.V. Carlson, L.F. Canto, S. Cruz-Barrios, M.S. Hussein and A.F.R. de Toledo Piza, Phys. Rev. C 592689 (1999).

[21] B.V. Carlson, M.S. Hussein, A.F.R. de Toledo Piza and L.F. Canto, Phys. Rev. C 60 014604 (1999).

[22] P.F. Bortignon and Ch. Dasso, Phys. Rev. C 56574 (1997).

[23] M.S. Hussein, A.F.R. de Toledo Piza and O.K. Vorov, Phys. Rev. C 59 R1242 (1999).

[24] R.T. de Paula et al, Phys. Rev. C 64064605 (2001)

[25] B.F. Bayman and F. Zardi, Phys. Rev. C59 2189 (1999).

[26] B.F. Bayman and F. Zardi, Phys. Rev. C 67017901 (2003).

[27] M. Goldhaber and E. Teller, Phys. Rev. 741046 (1948).

[28] D.H. Wilkinson, Physica 201039 (1956).

[29] D. M. Brink, Nucl. Phys. 4215 (1957)

[30] M. Abramowitz and I. A. Stegun (eds.), Handbook of Mathematical Functions (Dover, New York 1965)

[31] G. E. Brown and M. Bolsterli, Phys. Rev. Lett. 3472 (1959)

\section{Figure Captions}

Figure 1. (a) $V_{000-011}(\omega)$ for a bombarding energy of $10 \mathrm{~A} \mathrm{GeV}$ and an impact parameter of $12 \mathrm{fm}$. The peak at $\omega=0$ is narrower for the exact curve than for the longwavelength approximation because the exact curve shows the effect of dynamic damping. (b) $V_{000-011}(t)$ corresponding to the $V_{000-011}(\omega)$ of Figure 1a. The narrower $\omega$ dependence of the exact curve results in a broader $t$ dependence.

Figure 2. (a) The same as Figure 1a, but for an impact parameter of $50 \mathrm{fm}$. (b) The same as Figure 1b, but for an impact parameter of $50 \mathrm{fm}$.

Figure 3. Excitation probability of the state $\frac{1}{\sqrt{2}}\left[\Psi_{1}^{01}+\Psi_{-1}^{01}\right]$ as a function of $t$, for a bombarding energy of $10 \mathrm{~A} \mathrm{GeV}$ and an impact parameter of $200 \mathrm{fm}$. The Born approximation excitation probability is shown for comparison.

Figure 4. The same as Figure 3, except that the state is $\Psi_{0}^{01}$. The $t \rightarrow \infty$ prediction is $0.6 \times 10^{-5}$, compared to a Born approximation prediction of about $10^{-8}$.

Figure 5. The same as Figure 3, but for an impact parameter of $12 \mathrm{fm}$. 
Figure 6. The same as Figure 4, but for an impact parameter of $12 \mathrm{fm}$.

Figure 7. The asymptotic excitation probability of the state $\frac{1}{\sqrt{2}}\left[\Psi_{1}^{01}+\Psi_{-1}^{01}\right]$ as a function of impact parameter, for a bombarding energy of $10 \mathrm{~A} \mathrm{GeV}$.

Figure 8. The same as Figure 7 , except that the state is $\frac{1}{\sqrt{2}}\left[\Psi_{2}^{02}+\Psi_{-2}^{02}\right]$. Because of the importance of multistep processes in the excitation of this state, the Born approximation underestimates the excitation probability even at large impact parameter.

Figure 9. Cross-section for excitation of the state $\Psi_{0}^{01}$ as a function of the bombarding energy.

Figure 10. Cross-section for excitation of the state $\frac{1}{\sqrt{2}}\left[\Psi_{1}^{01}+\Psi_{-1}^{01}\right]$ as a function of the bombarding energy.

Figure 11. Total cross-section for excitation of the one-phonon states (the sums of the curves in Figures 9 and 10).

Figure 12. Cross-section for excitation of the state $\Psi_{0}^{02}$ as a function of the bombarding energy. The maximum Born cross-section is $0.038 \mathrm{mb}$.

Figure 13. Cross-section for excitation of the state $\frac{1}{\sqrt{2}}\left[\Psi_{1}^{02}+\Psi_{-1}^{02}\right]$ as a function of the bombarding energy.

Figure 14. Cross-section for excitation of the state $\frac{1}{\sqrt{2}}\left[\Psi_{2}^{02}+\Psi_{-2}^{02}\right]$ as a function of the bombarding energy.

Figure 15. Cross-section for excitation of the state $\Psi_{0}^{10}$ as a function of the bombarding energy. The maximum Born cross-section is $0.0006 \mathrm{mb}$.

Figure 16. Total cross-section for excitation of the two-phonon states (the sums of the curves in Figures 12, 13, 14, and 15). The maximum Born cross-section is $0.65 \mathrm{mb}$. 

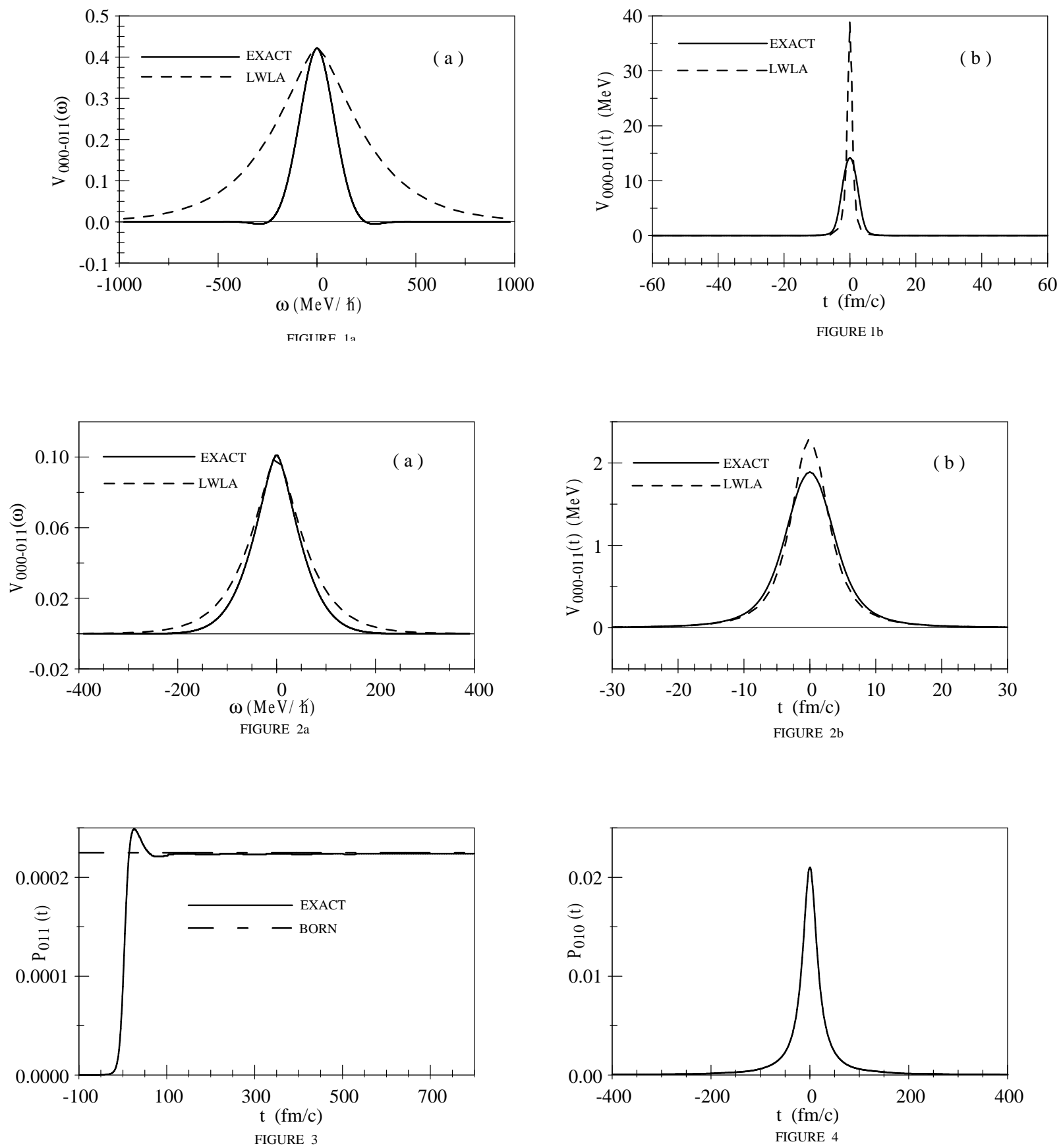

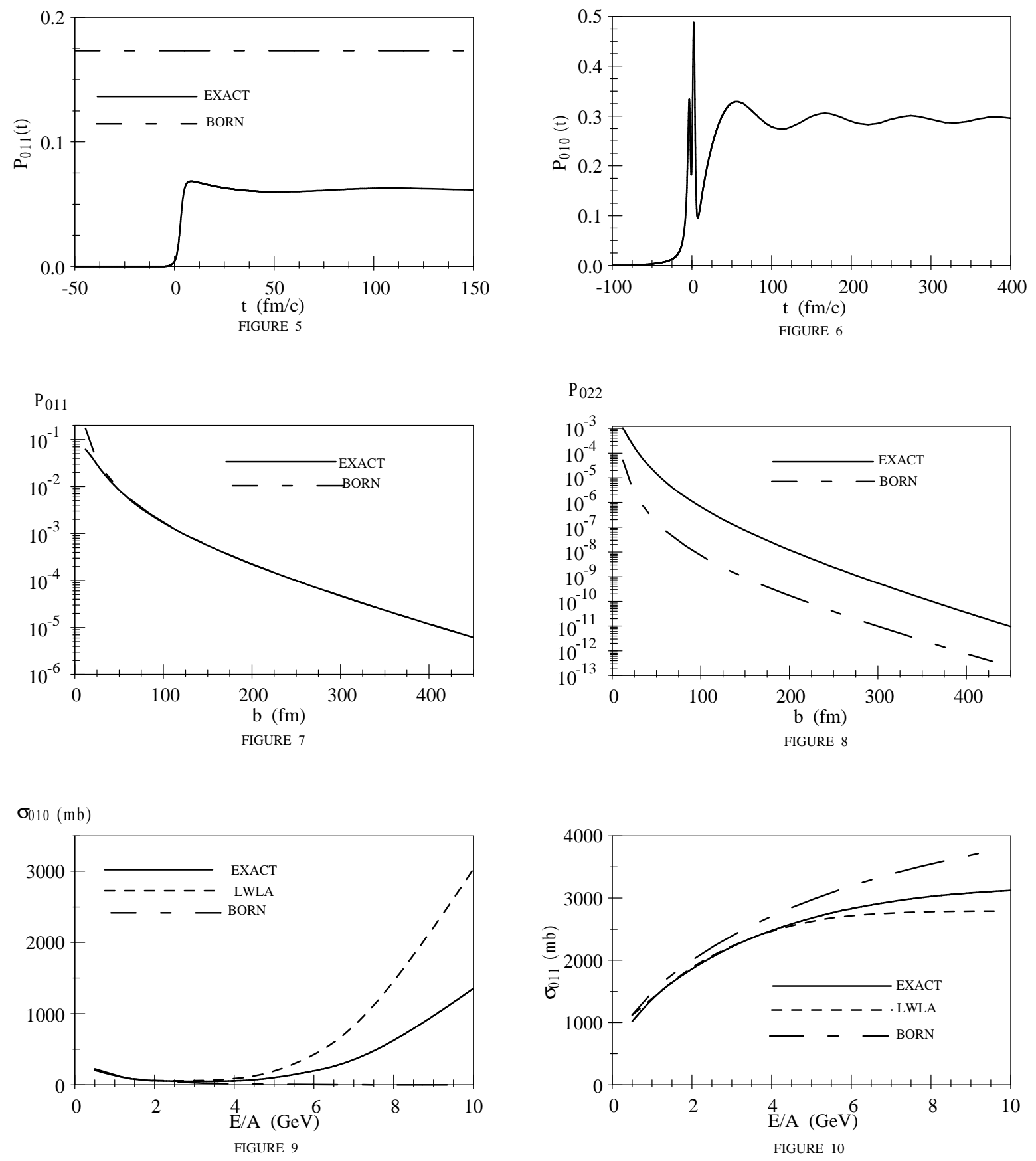

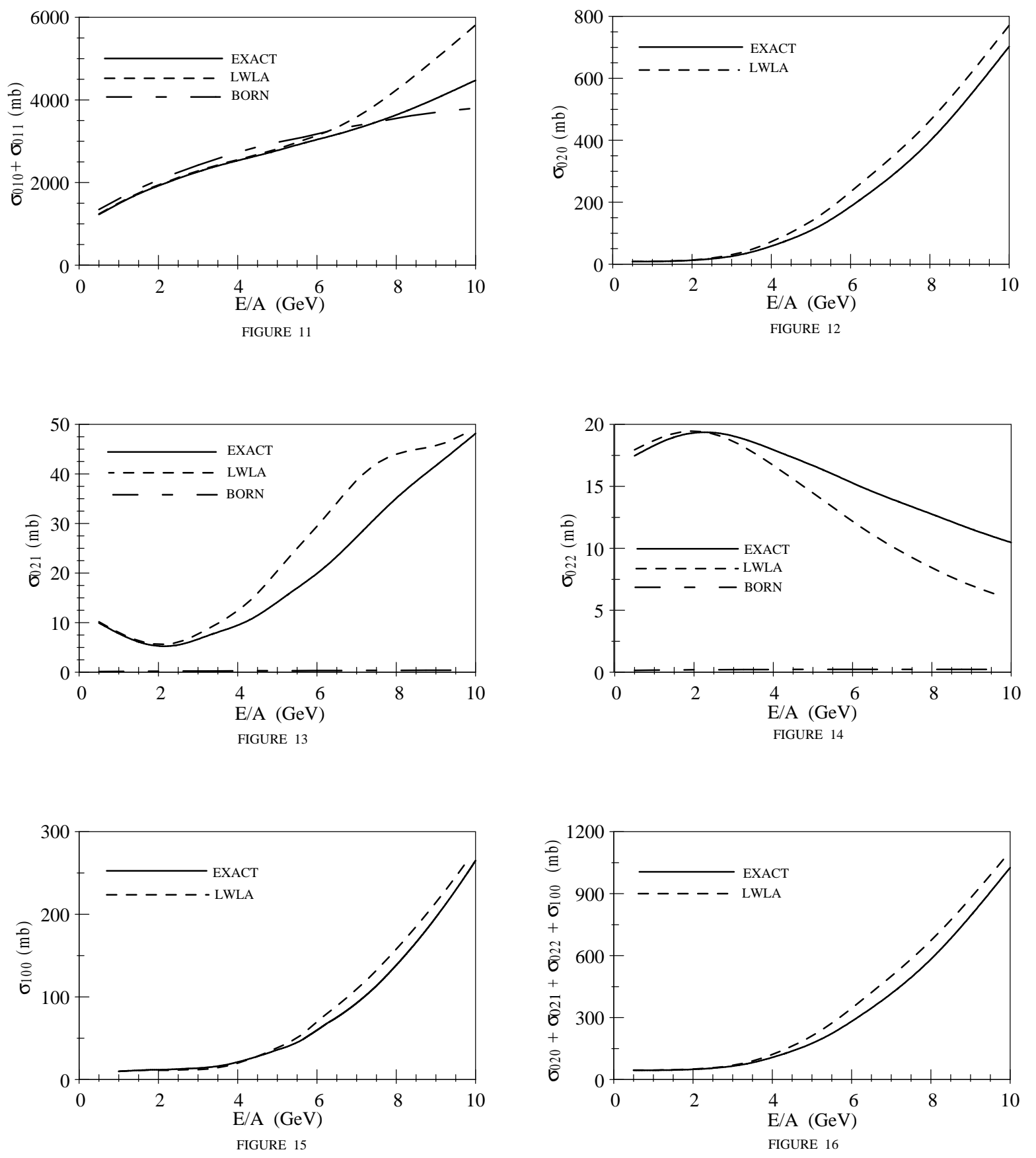